\title{
Teachers' Perspectives and Experiences of Social Emotional Learning in a Secondary School in Qatar
}

\author{
Noor Al-Wattary \\ College of Medicine, Qatar University, Doha-Qatar \\ E-mail: nalwattary@qu.edu.qa
}

\begin{abstract}
The purpose of this study was to explore teachers' knowledge and perspectives of social emotional learning (SEL) interventions in a Qatari school's settings by conducing 10 semi-structured interviews. Social emotional programs are still limited in Qatar and this study is one of the first studies that explored how teachers perceive SEL programs and what impact that they noticed on their students after SEL intervention. The results of the conventional content analysis yielded three main themes; SEL definition as perceived by teachers, SEL importance for students and SEL impact on social relations, academic performance and social behaviours. The study's findings offer a broader view of the primary teachers' views of social-emotional learning in the Qatari context. learning and effective practices that lead to the achievement of better outcomes within the Arabic and Qatari context. The results of this study are discussed in relation to the social context and the importance of teachers' perceptions as facilitators of SEL interventions in schools' settings.
\end{abstract}

Keywords: Social emotional learning (SEL), Teachers' perspectives, positive school environment

DOI: $10.7176 / \mathrm{JEP} / 12-36-01$

Publication date: December $31^{\text {st }} 2021$

\section{Introduction}

In the recent years, the interest in social emotional learning (SEL) started to increase and to continuously expand. There has been a substantial body of theory, research and practice that explored and evaluated the impact of SEL programs on students' wellbeing, social behaviors and academic performance (Humphrey, 2013, Durlak et al.,2011; Martin et al., 2017). SEL can be identified as the process " through which individuals learn and apply a set of social, emotional, behavioral, and character skills required to succeed in schooling, the workplace, relationships, and citizenship" (Jones et al., 2017, p. 12). In a more focused approach, the Collaborative for Academic, Social and Emotional Learning (CASEL) regards SEL as a "comprehensive multi-dimensional framework of the skills essential for successful social and emotional development” (Ross and Tolan, 2018, p. 1188).

Approaching the literature reveals that teachers, students and school contexts emerge as conflicting factors in the implementation process of SEL interventions. Teachers' perspectives and knowledge about SEL programs influence their role in the program's implementation process and may impede the success of its infusion (Dyson, Howley \& Shen, 2019). In relation, Humphrey, Barlow and Lendrum (2018) argue that teachers' perspectives about SEL intervention can influence the program implementation, efficacy and longevity. Therefore, evaluating teachers and school staff's perceptions of social emotional intervention is one of the main predictors of the program's outcomes (Wigelsworth, Humphrey \& Lendrum, 2012; McKown et al., 2013). In addition, a number of studies have called for more defined and sustained measurements of the effectiveness of SEL outcomes that include greater focus on measuring teachers' own perspectives of the SEL program as an important predictor of successful results (Humphrey, Barlow \& Lendrum, 2018; McKown et al., 2013; Pereira \& Marques-Pinto, 2018).

Humphries, Williams and May (2018) explored teachers' beliefs of the critical components and challenges of social emotional programs implemented in urban schools. The results revealed that the program's design, contextual relevance, support, and barriers were the emerged themes that can facilitate or hinder the program's success. Dyson, Howley and Shen (2019) explored teachers' perspectives of social emotional interventions in New Zealand primary school and found that the impact of SEL programs can be categorized into four main themes; positive interdependence, empowerment, self-management, self-awareness restorative conversations and circle time. Esen-Aygun and Sahin-Taskin (2017) investigated teachers' knowledge of SEL programs in the Turkish context and revealed that "that although most of the teachers had heard of the concept of social-emotional learning, they could not give a detailed explanation of it" (p.205). These various results indicate that teachers' knowledge and perspectives are important components of the social emotional interventions across cultures.

Teachers can act as the implementers of the SEL programs or the facilitators of the implementation process in general. Therefore, there is a need to understand teachers' perspectives in order to understand how does SEL work rather than focusing on does SEL work? (Wigelsworth et al., 2016). In particular, there is a lack of qualitative research exploring teachers' knowledge, perspectives and attitudes toward SEL interventions (Corcoran et al., 2018). Investigating these aspects can help in understanding and conceptualizing what makes effective and sustainable quality of SEL interventions on schools settings (Martínez, 2016).

When it comes to the Arabic context, studies that explore the interrelated factors that contribute to the SEL 
program's implementation and effectiveness is still limited. Specifically in Qatar, human development is one of the main pillars of the government vision. Within this vision, the Qatari government aspire to empower each individual with the required skills that enable him/her to achieve their future objectives and to contribute to their country's success. However, studies that investigate how teachers perceive, interact or conceptualize SEL interventions are still limited in Qatar. Therefore, this study was conducted as part of a larger case study project that aimed to explore the dynamics of the implementation process and the outcomes of a social emotional intervention in a government school in Qatar. This approach of exploring and evaluation teachers' knowledge about the implemented SEL program can create an in-depth contextual knowledge of what might work in the field. This knowledge and evidence-based outcomes can assist researchers and educational policymakers in designing the appropriate SEL interventions that serve students' social and emotional needs in the Arabic and in other similar contexts.

\section{Study context}

The primary objective of this study was to explore teachers' knowledge and perspectives of a social emotional intervention in a secondary public school for girls in Qatar. Social emotional learning in Qatar is relatively new concept that needs to be explored, evaluated and assessed to understand what can work in the field. Therefore, within this study, the aim was to capture teachers' subjective viewpoints about a social emotional intervention that was implemented in their school to deeply understand how they perceived the SEL intervention and what outcomes were achieved from their own perspectives. The study involved two main objectives; 1) to explore teachers' knowledge about SEL in general and 2) to evaluate their perceptions about the impact of the SEL program on their students. The SEL program in this study was a practitioner-designed, Islamic based social emotional intervention given to one group (intervention group). Interviews were conducted with the school's teachers who teach students in the intervention and the control groups (who did not participate in the program) to explore their perceptions and experiences with the SEL intervention. The question that guided this study were:

1. What is social emotional learning according to school teachers?

2. What is the impact of a social emotional programme from the teachers' and social staffs' perspectives in a Qatari girls'school?

\section{Methodology}

\subsection{Sample}

This research was conducted in a public secondary school for girls in Qatar. Public schools for girls in Qatar are managed and guided by female staff only. The program's sessions were mainly delivered by the program's instructor, who designed and implemented the SEL program. Teachers in this study did not deliver or participate in delivering the SEL intervention's sessions. Their role in this research was limited to participating in the study's interviews to explore their knowledge and perception of the program's impact on students. The sample consisted of 10 teachers, six of whom were Qataris, two Jordanian, one Palestinian and one Egyptian. Their subject areas were mathematics, English language, art, Arabic language and science. All the participating staff were qualified with a bachelor of art or science in their field of expertise. A Bachelor degree is the minimum accepted qualification in the Qatari public schools. Furthermore, all teachers had a minimum of three years of experience prior to joining the school. The participants' experiences in this study ranged from $5-15$ years.

\subsection{Data collection and analysis}

Semi-structured interviews were conducted individually with each participant using the Arabic language in an empty and quiet lecture room. In addition, due to the cultural and traditional perspectives, all staff refused to approve interviews' recording. Therefore, teachers' responses were recorded by hind writing during the interview to avoid losing any data.

The inductive approach was applied in the analysis of teachers' interviews. This inductive approach involved thematic analysis in searching for common themes, threads and categories that extend across the set of interviews (DeSantis \& Noel Ugarriza, 2000). Furthermore, this approach involved capturing themes that emerge directly from the data (Alshenqeeti, 2014). Humphries, Williams and May (2018) conceptualize thematic analysis as the process of reading and interpreting data by way of systematic classification to create themes that emerge from the data. Sandelowski and Leeman (2012) define themes as a coherent integration of the different components, responses and patterns of the data set in relation to the research question. Alshenqeeti (2014) describes the theme in the qualitative analyses as a descriptor that organizes a group of related and repeated ideas to unify the different concepts regarding the subject of inquiry. Therefore, theme determination can facilitate a comprehensive view by uncovering patterns related to participants' perspectives. In this study, the search for themes involved careful reading, classifying, comparing, and labelling the data to identify and collapse related patterns and major themes.

The inductive analysis approach aimed to capture participants' different experiences, viewpoints, suggestions, and impressions of the SEL program's impact in details. Thus, the analysis of all interviews was discussed based 
on the themes that emerged from the interviews data. As a result, three major themes emerged that guided the analysis process: definition of SEL programs, importance of SEL program and impact of the SEL programs on students. These three initial themes were related to the research questions.

Draucker et al. (2007) suggest that the initial analysis and interpretation of themes in the inductive data analysis leads to the development of categories. Hsieh and Shannon (2005) define categories as ideas that participants directly express in the text. As a result, for each theme, different categories were used to classify the findings in accordance with the interviews' results. These categories were the primary product of the analytical process and the descriptors of the themes.

\section{Results}

As mentioned above, within the data of teachers' interviews, three main themes have emerged; definition of SEL programs, importance of SEL program and impact of the SEL programs on students.

\subsection{Definition of SEL program}

The first emerged theme was how teachers defined SEL programs and it represents an "explanation of what is social emotional learning" based on teachers' perspectives. Therefore, all the meanings that teachers gave for social emotional learning as a concept were codified here. Teachers in this Qatari school did not experience or witness the implementation of SEL intervention in their school premises before. In addition, teachers represented limited knowledge of SEL interventions, mentioned that SEL programs are new to their school, and they did not experience the implementation of such interventions before.

I did not attend or participate in any workshop that is especially dedicated to students' social, emotional needs before. We have these skills as objectives, but no specific or real interventions were conducted in our school or in the other school that I worked with before. (Teacher 6)

Another teacher supported this feedback by stating that she did not participate or experience any of these interventions:

Social emotional learning as a program or an intervention is new to me, however, when I looked at the objectives of the program I am sure now that these interventions are so important to address our students' mental wellbeing and social skills needs. (Teacher 3)

In terms of available programs in Qatar, all teachers mentioned that there is a lack of these interventions:

We need social emotional interventions in our schools, and there are no available structured programs that address students' emotional needs from different dimensions; we really need these programs in Qatar. (Teacher 7)

\subsection{Importance of SEL programs}

For the theme of importance, two main categories involved; beliefs about SEL and the available interventions in Qatar. In terms of beliefs, all teachers emphasized the need for social emotional program in schools' settings, especially in the early adolescents' stage.

I think that these programs are especially needed for students at this age because students are experiencing a transitional period from childhood to the teenage stage, and they need to learn how to be responsible, respectful and successful candidates. (Teacher 9)

Another teacher emphasized the importance of empowering students with social emotional skills:

In this sensitive stage of life, equipping students with values and social skills is really needed-they act and interact with a social environment that influences their thinking approach, self-confidence, and social behaviours. Therefore, I am sure that social emotional intervention are needed and might result in significant improvements in students' social competencies. (Teacher 2)

One teacher viewed social emotional skills as a seed planted in students' heart that can grow and positively impact them in different situations:

I think that this program is like planting a seed and leave it to grow- the impact can be immediate, or longer time effect will be shown at different life stages. (Teacher 4)

In relation, another teacher explained the need for SEL programs for the Arabic community and based on the social and cultural norms:

Students need those programs for sure, the nature of life here, the busy parents, the social media influence and the interaction with other communities in our country are factors that emphasize the need for interventions based on Islamic, cultural and Arabic values that guide students' social and emotional skills, psychological needs and motivational competencies. (Teacher 9)

In more details, one teacher explained how the SEL interventions can improve students' wellbeing:

I am sure that when students' behaviours are better, their well-being will be better, which I noticed with my students. They look more optimistic, happier and motivated towards learning. (Teacher 7)

In a contrary perspective, one teacher mentioned that although the SEL interventions are essential, teachers might 
not be able to deliver or manage such interventions:

I am sure that SEL interventions are essential and needed for our kids and there is a lack in such programs in Qatar. However, we are already over-busy with our teaching and instruction tasks, so I don't think that we can implement such a program or have time for it. (Teacher 5)

\subsection{SEL impact on students}

Within the impact theme, three categories were emerged as the main themes according to teachers' perspectives. These results were based on teachers' perceived and noticed impact of the SEL intervention on their students. These subcategories included the impact on; teacher-student relationships, student-student relationships and students' classroom behaviours.

4.3.1 Teacher-student relationships

For the teacher-student relationships, teachers reported similar perspectives within this category. The majority of teachers believed that the SEL program increased students' awareness of the importance of respecting their teachers:

Ifeel that the program had a positive impact on students' social skills, such as respect; for instance, in one incident; I told a student (from the intervention group) who was faced with behavioral issues that I will call her mother-she immediately apologized - this reaction was a result of the respect lecture that the student attended in this program. (Teacher 3)

Another teacher confirmed these results by emphasizing that:

One of the major issues that I was always facing in school is students' disrespect. After the program, I noticed a significant and obvious change on students' attitudes toward me. When I ask them to participate in the classroom activities or to become quiet during the lesson, they immediately follow my instructions. (Teacher 10)

Another teacher described how she noticed a change in students' behaviours with her:

I think that students are calmer, happier and ready to listen and to follow their teachers' instructions. This is one of the main differences that I noticed in this regard in the intervention group students. (Teacher 6)

4.3.2 Student-student relationships

Teachers reported that the program had positively improved students' social relations with their peers. These results were observed and noted by teachers in the classroom settings. These relations, according to one teacher, involved dealing with others with respect and fewer bullying issues:

Students learned how to respect others, and I have noticed that in their classroom behaviours, they used to be more aggressive, less respective while now they are much better. I noticed that students are calmer, dealing with their peers in more positive attitudes and the number of conflict between students were significantly decreased. (Teacher 9)

Another teacher noticed how students started to share what they have learnt from the program with their peer students:

This experience is amazing you cannot imagine the level of impact that can spread to others, I noted a case in which one student from the intervention group talked to another in the control group about the program; as a result, students in the non-intervention group asked me if they can join. (Teacher 2)

Another teacher noticed similar outcomes and described how students were talking about the SEL program to their peers:

Students' interactions with the program were amazing. As a teacher for both the intervention and the control groups, I noticed that they convey the information and the lessons learned to other students from the other classrooms that did not participate in the program- that reflects the strong impact of the program. (Teacher 1)

In contrast to these feedbacks, one teacher emphasized that the period of the program was too short to notice any significant differences in students' relations outcomes:

I think that the program had a positive impact on some areas; however, I did not notice a difference in students' relationship with their peers for me as a teacher. I think that there is a need for more frequent and longer-lasting programs to achieve higher and more effective outcomes. (Teacher 4)

4.3.3 Classroom engagement

For the classroom engagement, teachers reported that students' participation in the classroom activities were significantly increased after they participated in the social emotional program. These results were confirmed by teachers' feedback in which they reported a significant difference in students' motivation, interest and participation in different classroom activities. For instance, one teacher mentioned that:

I noticed that students' interactions with the lessons and classroom activities are much better than before- they have the confidence now to participate- the motivation to answer and the dedication to be better, it was very obvious. (Teacher 7)

Another teacher supported this feedback by explaining the noticed positive change on students' willingness to participate in the classroom activities:

I noticed a difference in students' behaviours, specifically towards their academic engagement. Before this 
program, when I was asking questions in the classroom, the majority of students would not participate or share their thoughts; now, I noticed a significant improvement in their classroom engagement; they have the confidence to share their answers, thoughts and opinions regardless of whether they are right or wrong. (Teacher 6)

In relation, one teacher emphasized that the program encouraged less active students to participate in the classroom's activities:

The program effectively encouraged the majority of self-isolated and less-active students to be part of the classroom activities, participate, and express their feelings. I feel that students are better now in terms of their self-confidence levels, they are more responsible and that is reflected in their classroom behaviours. (Teacher 2) These outcomes were similar to another feedback that explained students' willingness to be successful candidates in the future:

One of my students informed me how the program had influenced her thinking of the taste of success, the value of achievement and the plans to reach dreams, and how she is planning to get a bachelor's degree and a master and a PhD as well. This feedback describes the impact of SEL program on developing students' inner-thinking and motivation towards achievement. (Teacher 10)

In this regard, another teacher mentioned outcomes related to students' motivation:

In terms of classroom behaviours, I noticed that students have positively changed to have better motivation levels, more interactions and less behavioural issues with the teacher and fellow students. Furthermore, students reflected that they are willing to learn, they ask, share, participate and contribute to the different activities. (Teacher 8) In contrast to these findings, one teacher reported that:

I am sure that the program was a very good experience for each student, however, in terms of academic and classroom engagement, I think that students needed more sessions that focus on these dimensions. Thus, I do not think that I had observed significant changes in students' classroom behaviour or academies engagement after the program. (Teacher 6)

Another teacher supported this feedback by stating that:

The period of the program was so short, so I do not think that we can notice a change in this short time. (Teacher 5)

\section{Discussion}

Teachers in the present study did not have prior experiences with SEL interventions in the Qatari schools' settings; however, they had strong beliefs about these interventions' importance. In addition, teachers tried to explain their understanding of SEL interventions are, the objectives of these interventions, and whether these interventions can be conducted in schools' settings.

These results are consistent with Esen-Aygun and Sahin-Taskin's (2017) findings, which reported that most of the teachers' knowledge about SEL programs was very limited and they were not aware of SEL programs implemented in their schools. In addition, other previous research similarly showed that teachers did not have enough knowledge about socicial emotional learning, and their experiences were inadequate about SEL interventions (Buchanan et al.,2009; Zakrzewski, 2013) However, in this study, all teachers mentioned the need for implementing SEL programs in schools, especially to serve students' needs at this age. They argued that there is a lack of school-based interventions that serve students' developmental needs through the adolescents' stage of life in Qatar.

In addition, teachers reported that social emotional programs are crucially needed for this generation as they are exposed to different attractions through the social media apps and websites. These different social media sources can expose different values, experiences and challenges that might influence their way of thinking and their mental wellbeing levels. Thus, according to teachers, there is a need to consider SEL initiative as an approach that equips students emotional, social and different life skills that can improve students' relationships with others, schooling engagement and relationships with their teachers. Furthermore, teachers' responses to the interviews showed that SEL interventions need to be related to the religious, cultural and social environment in which students live to address their social-emotional development. These results are related to many previous findings that explored teachers' and school staff's perceptions of the impact of SEL interventions. Triliva and Poulou. (2006) reported in their study that teachers believed that students need a solid foundation and morals to address their social and emotional competencies. Buchanan et al. (2009) revealed similar results that emphasized teachers' strong sense of responsibility to support student's social and emotional abilities. They reported in their study that examined teachers' knowledge, perceptions and practices regarding SEL interventions that many teachers believed that SEL programs are important to serve their students' social and emotional needs. In addition, from a different cultural vantage point Van Huynh, Tran-Chi and Nguyen (2018) revealed that all the Vietnamese teachers in their study were aware of the necessity of social emotional interventions in addressing students' emotional needs.

After the SEL program, teachers noticed significant changes in students' behaviours in the intervention group. These changes can be categorized within three main dimensions; classroom behaviours, teacher-student relationships and student-student relationships. In terms of the first dimension, the classroom behaviours, teachers 
mentioned in their interviews that they witnessed significant changes in students' classroom engagement, collaboration and participation in the sessions' activities. Students started to participate more in the sessions' activities and shared their thoughts and answers and demonstrated dedication towards achievement. A possible explanation for this result is that when students' self-confidence, self-realization and wellbeing levels were increased, their willingness to participate in different activities were also increased. They demonstrated motivation towards achieving better results in their interviews, and as a result, they started to apply what they learned into practice. These results are related to Gage and Thomas (2019) findings in which teachers perceived a notable difference in students' self-regulation and classroom engagement. Furthermore, Borner (2020) revealed that, based on teachers' perceptions, social emotional interventions positively affected student behaviours in classroom settings and their relationships with teachers and their peer students.

In terms of the second dimension; teacher-student relationship, teachers mentioned that they noticed differences in students' approach with teachers and school staff. In some cases, students used to be less respectful, undertaking more arguments and avoiding the teacher's advice and comments. After the program, teachers observed significant changes in the majority of students' behaviours. They noticed that students are focusing on their learning, willing to listen to their teachers and demonstrating respect skills. These results are similar to many previous studies that consider warm classroom teacher-student relationships as a major component in promoting effective learning, positive social emotional competencies and wellbeing levels (Schonert-Reichl, 2017; Wigelsworth, Humphrey \& Lendrum, 2012). Therefore, these results suggest a more holistic approach in investigating the impact of SEL interventions that incorporate teachers as well as students to explore the outcomes from different dimensions.

The third dimension of teachers' interviews revealed that student-student relationships were improved after participating in the SEL program. Teachers noticed a decrease in students' behavioral issues with their peers. They noticed that, even students were having behavioral issues, they were calm, willing to solve issues and hoping to avoid any future issues with their peers. In relation, some teachers noticed a significant decrease in the number of students' behavioral reports after the SEL program from those students who took part in the intervention. These results indicate that, despite the short time of implementation, the social emotional program in this study had a positive impact on students' social and classroom behaviours. In addition, these results reveal that the SEL program's outcomes can be noticed during the implementation process as well as after the full implementation of the program. The social emotional program in this study had improved students' social emotional skills from multiple dimensions. Students started to build better relationships with their peers and teachers, they applied what they learned into practice, and they started to develop the sense of belonging to the school's educational environment. In addition, teachers noticed these improvements during and after the program's implementation by noticing significant decrease in students' social behavioral issues with their peers.

Although this feedback was based on a short-term effect, these results indicate that there was a positive outcome of this SEL intervention on students' social relations with others. This aligns with theory and research on the impact of the stability and security of the student-relationship on students' wellbeing and emotional regulation. Hyson (2004) emphasized the need for secure, positive and nurturing student-teacher relationships to form caring attachments and trust with their teachers. Triliva and Poulou (2006) argue that positive student-teacher relationships can foster students' sense of wellbeing by enabling them to feel safe within their learning environment. As a result, students within a safe, friendly and secure environment can learn to become more motivated, collaborative and empathetic to achieve personal and academic goals (Wigelsworth, Humphrey \& Lendrum, 2012).

In contrast, two teachers mentioned that they did not notice a difference in students' behaviours or motivation towards learning in her classroom. One teacher related these results to the relatively short period of SEL implementation in which students did not have adequate time to develop different social and emotional competencies. In addition, the second teacher emphasized the need for more SEL sessions to evaluate any possible impact on students. These results are related to many studies in which teachers did not notice a difference in students' behaviours or classroom engagement. Harrington et al. (2020) revealed that teachers did not report a significant change in students' behaviour after participating in a social emotional intervention. In this regard, Buchanan et al. (2009) revealed in their study that examined teachers' knowledge, perceptions, and practices regarding SEL that teachers believe that SEL is important; however, the academic demand might decrease the effective implementation of SEL interventions and application in school settings. In this study, the SEL programme was implemented within a short period of time by the program's instructor, therefore, more longitudinal data that involve teachers in the implementation process is essential to assess these issues in more details.

\section{Limitations}

The limitations of this study are represented by the short-term of evaluation of the program. In addition, all qualitative research is inherently limited by the fact that its findings cannot be generalized to people who were not included in the study sample. With regard to future research, three types of studies are suggested to be considered. The first of these would be to apply similar SEL interventions with larger samples of students and teachers to get 
more perspectives that can be generalized to other similar contexts. The second study could involve teachers in the implementation of SEL intervention to explore their experiences, perspectives and knowledge of the program as an integrated process. And finally, based upon the findings from this study and related previous studies, it is suggested that more longitudinal research to be conducted to investigate the impact of SEL interventions over longer periods of time.

\section{Conclusion}

This study sought to evaluate the impact of the social emotional program based on teachers' perspectives by involving teachers who teach students in an intervention and a control group of a social emotional intervention. The study is one of the first attempts to explore SEL interventions' dynamics in the Qatari context. Teachers' perceptions, understandings and feedback of the knowledge, importance and impact of SEL were moderately correlated. This study provides evidence about the outcomes of the SEL program based on teachers' perspectives and experiences with their students. Furthermore, these results indicate the need for SEL programs in Qatar to improve students' classroom engagement and relationships with their teachers and their peers

\section{References}

Alshenqeeti, H. (2014). Interviewing as a data collection method: A critical review. English linguistics research, 3(1), 39-45.

Borner, L. T. (2019). Teachers' Perceptions regarding the Implementation of Social-Emotional Learning in Title I Schools (Doctoral dissertation, Houston Baptist University).

Buchanan, R., Gueldner, B. A., Tran, O. K., \& Merrell, K. W. (2009). Social and emotional learning in classrooms: A survey of teachers' knowledge, perceptions, and practices. Journal of Applied School Psychology, 25(2), 187-203.

Buchanan, R., Gueldner, B. A., Tran, O. K., \& Merrell, K. W. (2009). Social and emotional learning in classrooms: A survey of teachers' knowledge, perceptions, and practices. Journal of Applied School Psychology, 25(2), 187-203.

Corcoran, R. P., Cheung, A. C., Kim, E., \& Xie, C. (2018). Effective universal school-based social and emotional learning programs for improving academic achievement: A systematic review and meta-analysis of 50 years of research. Educational Research Review, 25, 56-72.

DeSantis, L., \& Ugarriza, D. N. (2000). The concept of theme as used in qualitative nursing research. Western journal of nursing research, 22(3), 351-372.

Draucker, C. B., Martsolf, D. S., Ross, R., \& Rusk, T. B. (2007). Theoretical sampling and category development in grounded theory. Qualitative health research, 17(8), 1137-1148.

Durlak, J. A., Weissberg, R. P., Dymnicki, A. B., Taylor, R. D., \& Schellinger, K. B. (2011). The impact of enhancing students' social and emotional learning: A meta - analysis of school - based universal interventions. Child development, 82(1), 405-432.

Dyson, B., Howley, D., \& Shen, Y. (2019). Teachers' perspectives of social and emotional learning in Aotearoa New Zealand primary schools. Journal of Research in Innovative Teaching \& Learning.

Esen-Aygun, H., \& Sahin-Taskin, C. (2017). Teachers' Views of Social-Emotional Skills and Their Perspectives on Social-Emotional Learning Programs. Journal of Education and Practice, 8(7), 205-215.

Harrington, Ellie M., Shaina D. Trevino, Sheila Lopez, and Nicole R. Giuliani. "Emotion regulation in early childhood: Implications for socioemotional and academic components of school readiness." Emotion 20, no. 1 (2020): 48.

Hsieh, H. F., \& Shannon, S. E. (2005). Three approaches to qualitative content analysis. Qualitative health research, 15(9), 1277-1288.

Humphrey, N., Barlow, A., \& Lendrum, A. (2018). Quality matters: Implementation moderates student outcomes in the PATHS curriculum. Prevention Science, 19(2), 197-208.

Humphrey, R. H. (2013). The benefits of emotional intelligence and empathy to entrepreneurship. Entrepreneurship Research Journal, 3(3), 287-294.

Humphries, M. L., Williams, B. V., \& May, T. (2018). Early childhood teachers' perspectives on social-emotional competence and learning in urban classrooms. Journal of applied school psychology, 34(2), 157-179.

Hyson, M. (2004). The emotional development of young children: Building an emotion-centered curriculum. Teachers College Press.

Jones, S. M., Barnes, S. P., Bailey, R., \& Doolittle, E. J. (2017). Promoting social and emotional competencies in elementary school. The future of children, 49-72.

Martin, A. J., Collie, R. J., \& Frydenberg, E. (2017). Social and emotional learning: Lessons learned and opportunities going forward. In Social and Emotional Learning in Australia and the Asia-Pacific (pp. 459471). Springer, Singapore.

Martínez, L. (2016). Teachers' voices on social emotional learning: Identifying the conditions that make 
implementation possible.

McKown, C., Allen, A. M., Russo-Ponsaran, N. M., \& Johnson, J. K. (2013). Direct assessment of children's social-emotional comprehension. Psychological Assessment, 25(4), 1154.

Pereira, N. S., \& Marques-Pinto, A. (2018). Development of a social and emotional learning program using educational dance: A participatory approach aimed at middle school students. Studies in Educational Evaluation, 59, 52-57.

Ross, K. M., \& Tolan, P. (2018). Social and emotional learning in adolescence: Testing the CASEL model in a normative sample. The Journal of Early Adolescence, 38(8), 1170-1199.

Sandelowski, M., \& Leeman, J. (2012). Writing usable qualitative health research findings. Qualitative health research, 22(10), 1404-1413.

Schonert-Reichl, K. A. (2017). Social and emotional learning and teachers. The future of children, 137-155

Triliva, S., \& Poulou, M. (2006). Greek teachers' understandings and constructions of what constitutes social and emotional learning. School Psychology International, 27(3), 315-338.

Triliva, S., \& Poulou, M. (2006). Greek teachers' understandings and constructions of what constitutes social and emotional learning. School Psychology International, 27(3), 315-338.

Van Huynh, S., Tran-Chi, V. L., \& Nguyen, T. T. (2018). Vietnamese Teachers' Perceptions of Social-Emotional Learning Education in Primary Schools. European journal of contemporary education, 7(4), 874-881.

Wigelsworth, M., Humphrey, N., \& Lendrum, A. (2012). A national evaluation of the impact of the secondary social and emotional aspects of learning (SEAL) programme. Educational Psychology, 32(2), 213-238.

Wigelsworth, M., Humphrey, N., \& Lendrum, A. (2012). A national evaluation of the impact of the secondary social and emotional aspects of learning (SEAL) programme. Educational Psychology, 32(2), 213-238.

Wigelsworth, M., Lendrum, A., Oldfield, J., Scott, A., Ten Bokkel, I., Tate, K., \& Emery, C. (2016). The impact of trial stage, developer involvement and international transferability on universal social and emotional learning programme outcomes: A meta-analysis. Cambridge Journal of Education, 46(3), 347-376.

Zakrzewski, V. (2013). Why teachers need social-emotional skills. Greater Good Magazine, 13. 